\title{
Spectral Properties of the Attractive Hubbard Model
}

\author{
M. Letz, R. J. Gooding \\ Dept. of Physics, Queen's University, Kingston, Ontario, Canada K'L 3N6
}

(October 25, 2018)

\begin{abstract}
Deviations from Fermi liquid behavior are well documented in the normal state of the cuprate superconductors, and some of these differences are possibly related to pre-formed pairs appearing at temperatures above $T_{c}$. In order to test these ideas we have investigated the attractive Hubbard model within a self-consistent, conserving ladder approximation. In this version of the theory, no feature is present which can be related to the pseudo gap found in the high- $\mathrm{T}_{c}$ materials. Further, the interactions between two-particle bound states change the physics of the superconducting instability in a profound fashion, and lead to a completely different phenomenology that one predicts based on the non-self-consistent version of the same theory.
\end{abstract}

PACS: $71.27 .+\mathrm{a}$ 
Numerous recent experiments (e.g., ARPES, optical, neutron scattering, NMR) have shown that in the high $\mathrm{T}_{c}$ cuprate superconductors a so-called pseudo-gap is present [1]; this is further supported by the extensive theoretical work of Randeria and collaborators [2]. This has led to proposals that electron pairs form at temperatures well above the superconducting transition temperature. However, possibly due to phase fluctuations, a macroscopic phase coherent wave function is not formed, so superconductivity is not encountered until lower temperatures [3].

This physics motivates our study of the attractive Hubbard model. The Hamiltonian for this system is

$$
H=-t \sum_{\langle i j\rangle, \sigma}\left(c_{i, \sigma}^{\dagger} c_{j, \sigma}+\text { h.c. }\right)-|U| \sum_{i} n_{i, \uparrow} n_{i, \downarrow}
$$

where the lattice sites of a 2-D square lattice are labeled by $\{i\}$, the lattice fermion operators are denoted by $c_{i, \sigma}$, and neighbouring sites are represented by $\langle i j\rangle$. For any nonzero $|U|$, two-particle bound states appear, and are physically related to a pair of electrons lowering the system's energy when they exist on the same lattice site. This is certainly the simplest example of a model Hamiltonian which allows for one to study the interactions between such electron pairs.

We employ the Brueckner-Hartree-Fock theory (or ladder approximation) which neglects all crossing diagrams and which is therefore valid only at low electronic densities. A preformed pair manifests itself in this theory as a two-particle bound state. In the non-self-consistent version of the theory such a pair of electrons always has an infinite lifetime.

By analyzing a fully self-consistent formulation of this theory, we are able to investigate preformed pairs with arbitrary lifetime. That is, if one solves this problem in this approximation, one includes pair-pair interactions. Haussmann has argued [4] (when he investigated a threedimensional continuum model with the same physics) that the interactions between pairs will be, in first order, of a repulsive nature. This leads to the dissociation of the pairs causing its finite lifetime.

The equations for the Green's function $(G)$, pair susceptibility $(\chi)$, four-leg vertex function $(\Gamma)$, and self energy $(\Sigma)$, in a conserving approximation are well known:

$$
\begin{aligned}
G\left(\mathbf{k}, i \omega_{n}\right) & =\left(G_{0}\left(\mathbf{k}, i \omega_{n}\right)^{-1}-\Sigma\left(\mathbf{k}, i \omega_{n}\right)\right)^{-1} \\
\chi\left(\mathbf{K}, i \Omega_{n}\right) & =\frac{-1}{N \beta} \sum_{m, \mathbf{k}} G\left(\mathbf{K}-\mathbf{k}, i \Omega_{n}-i \omega_{m}\right) G\left(\mathbf{k}, i \omega_{m}\right) \\
\Gamma\left(\mathbf{K}, i \Omega_{n}\right) & =|U| /\left(1+|U| \chi\left(\mathbf{K}, i \Omega_{n}\right)\right) \\
\Sigma\left(\mathbf{k}, i \omega_{n}\right) & =\frac{1}{N \beta} \sum_{m, \mathbf{q}} \Gamma\left(\mathbf{k}+\mathbf{q}, i \omega_{n}+i \omega_{n}\right) G\left(\mathbf{q}, i \omega_{m}\right)
\end{aligned}
$$

where the wave vectors and Matsubara frequencies have their usual meaning. In a non self-consistent theory, one replaces the full Green's functions $G$ in Eqs. $(3,5)$ with the noninteracting Green's functions $G_{0}$. The usual Thouless criterion begins with the non self-consistent theory and identifies an instability [5] of the normal state in terms of poles of the pair susceptibility (in a weakly interacting 3-D system, the Thouless criterion becomes identical to the equation which determines $\mathrm{T}_{c}$ in the BCS theory). The changes in this phenomenology that arise when one includes pair-pair interactions may be an important component of the high $\mathrm{T}_{c}$ problem.

For full self consistency, this set of equations has to be solved iteratively. Since such solutions are difficult and time consuming to obtain, we have investigated a simple approximation that allows for extensive numerical investigations of the resulting equations. To be specific, during the first step of the iteration process leading to self consistency, we make an approximation for the pair susceptibility as being equal to the $\mathbf{k}$-average (denoted from now on as over-lined quantities, e.g. $\bar{\Gamma}$ ) of the noninteracting pair susceptibility. We only calculate the $\mathbf{k}$-averaged pair susceptibility during subsequent iterations to self consistency. This leads to the following expressions

$$
\begin{aligned}
& \bar{\Gamma}\left(i \Omega_{n}\right)=\frac{1}{N} \sum_{\mathbf{K}} \Gamma\left(\mathbf{K}, i \Omega_{n}\right) \approx|U| /\left(1+|U| \bar{\chi}\left(i \Omega_{n}\right)\right) \\
& G\left(\mathbf{k}, i \omega_{n}\right) \approx\left(G_{0}\left(\mathbf{k}, i \omega_{n}\right)^{-1}-\bar{\Sigma}\left(i \omega_{n}\right)\right)^{-1}
\end{aligned}
$$

Besides the computational simplicity of this approach, one important additional advantage of this technique arises from the ease with which low temperatures may be reached for two and higher dimensional systems. Other methods have great difficulty reaching the low temperatures for which superconducting properties may become apparent, potentially making our approach quite valuable.

Our k-averaged approximation becomes accurate in any of the following limits: Quite trivially, (I) At large temperatures all correlations are thermally washed out, and for small bandwidths one obtains the atomic limit. In both cases, k-dispersion of the pair susceptibility is irrelevant. (II) For large U, $\Gamma$ is basically determined by the pole of the bound state at $\chi=1 / U$ which means that a weakly dispersive two particle bound state is well separated from the continuum. So, in this case the average over $\mathbf{k}$-space is a good approximation, since the bound state below the continuum is now replaced by it's k-average. (III) Probably the most interesting limit in which our approximation is valid is that of large spatial dimensions. It was argued by Vollhardt and collaborators 6 that for a system with large dimensions the k-dispersion of the self-energy vanishes. Such an approximation gives several results which are also valid in lower dimensions. This limit gives us a tool to compare our final result with well known results obtained from the 
Kondo-impurity problem. This is due to the possibility of mapping the attractive Hubbard model, via a particlehole transformation, onto a positive U Hubbard model in a magnetic field. The repulsive Hubbard model, in the limit of large spatial dimensions, becomes equivalent to the Kondo impurity problem [7]. We will discuss more fully the regimes of validity of this approximation in a future publication.

We begin by showing that for a one dimensional system the $\mathbf{k}$-average method correctly reproduces the physics found in a fully self-consistent, conserving theory even in the intermediate coupling regime. In Fig. 11 we have plotted the variation of electron density with temperature for $|U|$ being equal to the bandwith for $\mu=-t$ (note that the free electron band extends from $-2 t$ to $2 t$ ). This quantity was calculated firstly for the self-consistent, conserving theory obtained from the $\mathbf{k}$-average method, and then from the full theory where $\mathbf{k}$-dispersion was taken into account. The latter work involved 150 points along the imaginary frequency axis and 40 real-space lattice points - we found that these numbers gave fully converged results. In both cases the electron density decreases monotonically with temperature, and there is no sign of a divergence (or strong increase) of the particle number.

We note that this behaviour is in direct contrast to results obtained from non self-consistent calculations. In one and two dimensions, for low temperatures, the system is always unstable towards a condensation into an infinite lifetime two-particle bound state. For two dimensions this was demonstrated for a non self-consistent, non conserving theory by Schmitt-Rink, et al. [8], and we [9] have recently shown similar physics is encountered when a fully conserving theory is used. However, the self-consistent theories, at least to the minimum temperatures that we have been able to access, do not show similar physics.

In this brief report we want to focus on only one aspect of the numerical results which we have generated from our $\mathbf{k}$-averaged treatment of the self-consistent, conserving treatment of the attractive Hubbard model. In Fig. 2 we show the density of states $\mathrm{A}(\omega)$ at different temperatures for a two-dimensional square lattice, again taking $|U|$ to be equal to the bandwidth, at a low electron density $n=0.3$ ( $n=0.5$ corresponds to half filling). We have also shown the results that follow from other approximations.

The non self-consistent conserving approximation leads to a large fraction of the density of states being shifted to a peak below the one particle continuum this peak is due to the infinite lifetime two-particle bound state. Between this peak and the one particle continuum lies a large gap, and this gap has been suggested by some to be the origin of the pseudo gap.

However, our self-consistent, conserving, k-averaged calculation shows that the gap mentioned above disappears entirely, and that the only remnant from the two particle bound state is a flat, strongly lifetime broadened shoulder below the continuum. Further, instead of a gap, the density of states becomes a small maximum at the chemical potential, and with decreasing temperature this maximum increases. The existence of such a maximum can be understood via the analogy of the attractive Hubbard model with the repulsive Hubbard model which in large spatial dimensions can be mapped onto a Kondo impurity model as mentioned above. In such problems a local maximum of the one particle density of states also occurs, as seen, e.g., by Georges et al. 10, who investigated the repulsive Hubbard model by using an iterated perturbation theory.

We note that recent work for a $d$-wave pairing interaction [11] has found similar results, but has been interpreted quite differently.

In conclusion, we have demonstrated the possibility of solving the attractive Hubbard model in two dimensions in the self-consistent, conserving ladder approximation by performing a numerically advantageous $\mathbf{k}$-average. Due to self-consistency, we include pair-pair interactions which hinder the formation of an infinite lifetime bound state. In this way, the Thouless criterion is altered. Further, evidence for a pseudo-gap in the density of states is not obvious.

We thank Frank Marsiglio for helpful comments. This work was supported by the DFG (Deutsche Forschungsgemeinschaft), and the NSERC of Canada.

[1] See, e.g., contributions in the Proceedings of the 10th Anniversary $H_{c}$ Workshop on Physics, Materials, ed. by B. Batlogg, C. W. Chu, et al. World Scientific, (1996).

[2] See e.g. M. Randeria, in Bose Einstein Condensation Ed. A. Griffin et al. (Cambridge University Press, 1995)

[3] V. J. Emery and S. A. Kivelson, Nature 374, 434 (1995).

[4] R. Haussmann, Z. Phys. B 91, 291 (1993).

[5] R. D. Mattuck, A Guide to Feynmann Diagrams in the Many-Body Problem, p. 224 (McGraw-Hill, 1967).

[6] P. G. J. van Dongen, and D. Vollhardt, Phys. Rev. Lett. 65, 1663 (1990).

[7] T. Schork, S. Kehrein, M. Ulmke (private communication).

[8] S. Schmitt-Rink, C. M. Varma, and A. E. Ruckenstein, Phys. Rev. Lett. 63, 445 (1989).

[9] M. Letz, F. Marsiglio, R. J. Gooding (in preparation).

[10] A. Georges and W. Krauth, Phys. Rev. B 48, 7167 (1993).

[11] J. R. Engelbrecht, A. Nazarenko, M. Randeria, and E. Dagotto, cond-mat/9705166. 


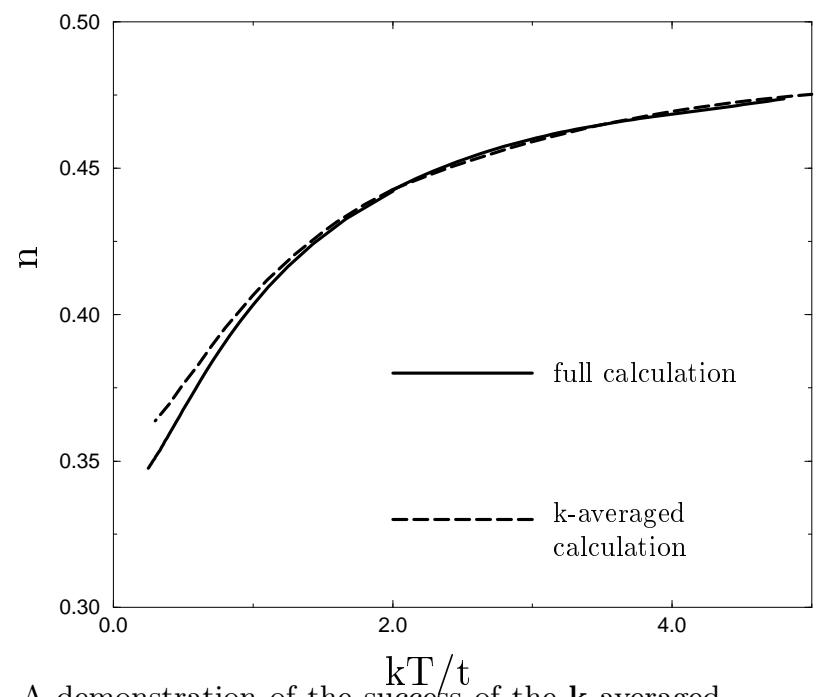

FIG. 1. A demonstration of the success of the $\mathbf{k}$-averaged method for a one-dimensional system. Here, $|U| / t=4$, namely the Hubbard energy is equal to the bandwidth, and the chemical potential is fixed to be $\mu / t=-1$. Then, the electron density $n$ is calculated as a function of temperature. The solid line shows the "exact" calculation for the self-consistent, conserving approximation, and the $\mathbf{k}$-averaged result is shown by the dashed line. All energies are given in units of $t$.

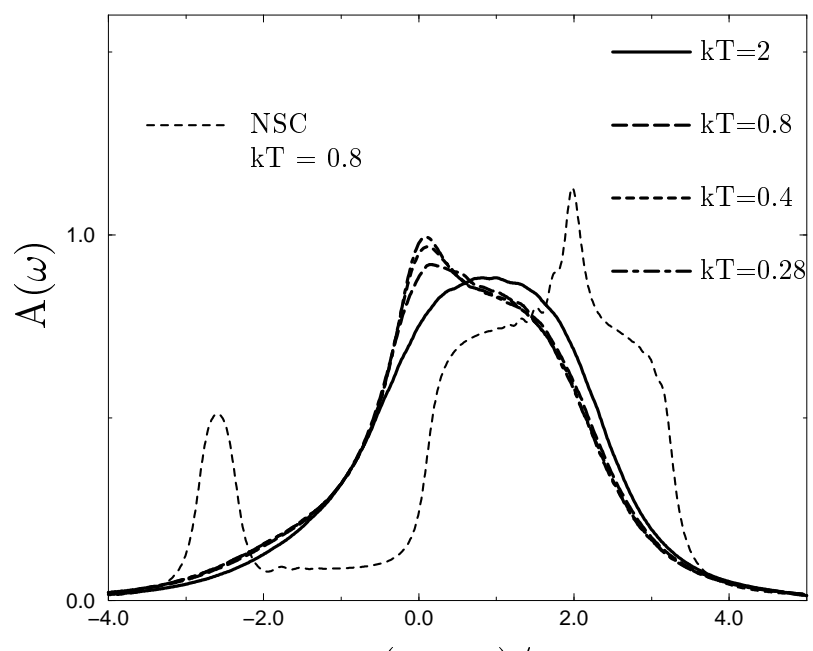

FIG. 2. The density of states $A(\omega)$ that $) / t$ two-dimensional system with $\mathrm{n}=0.3$ and $|U| / t=8$ for four different temperatures. Note that the density of states develops a maximum at the chemical potential when the temperature is decreased. 